\title{
Statistics of Coulomb Blockade Peak Spacings within the Hartree-Fock Approximation
}

\author{
Avraham Cohen ${ }^{1}$, Klaus Richter ${ }^{2}$, and Richard Berkovits ${ }^{1}$ \\ ${ }^{1}$ The Minerva Center for the Physics of Mesoscopics, Fractals and Neural Networks, Department of Physics, \\ Bar-Ilan University, 52900 Ramat-Gan, Israel \\ ${ }^{2}$ Max-Planck-Institut für Physik komplexer Systeme, Nöthnitzer Strasse 38, 01187 Dresden, Germany
}

(April 23, 2022)

\begin{abstract}
We study the effect of electronic interactions on the addition spectra and on the energy level distributions of two-dimensional quantum dots with weak disorder using the self-consistent HartreeFock approximation for spinless electrons. We show that the distribution of the conductance peak spacings is Gaussian with large fluctuations that exceed, in agreement with experiments, the mean level spacing of the non-interacting system. We analyze this distribution on the basis of Koopmans' theorem. We show furthermore that the occupied and unoccupied Hartree-Fock levels exhibit Wigner-Dyson statistics.
\end{abstract}

PACS numbers: 72.10.Fk, 73.20.Dx

Introduction - It has recently been established that studying the low temperature fluctuations of the conductance through quasi-isolated nanostructures is an excellent tool for probing electronic interactions [1]. In such small devices called artificial atoms [2] or quantum dots it is possible to control and successively vary the total number of electrons from zero [3] to a few hundreds [ [ [7]. When coupled weakly to leads, the conductance of the dots is vanishingly small between pronounced peaks because of Coulomb blockade [8]. By tuning a gate voltage connected to the dot a conductance peak is observed whenever the ground state energy $E_{G}(n)$ of the dot containing $n$ electrons becomes degenerate with the energy of the dot with $n+1$ electrons. At almost zero drain to source voltage this situation is expressed as $\mu_{n} \equiv E_{G}(n+1)-E_{G}(n)=\mu_{\text {Lead }}$, where $\mu_{\text {Lead }}$ and $\mu_{n}$ is the chemical potential of the leads and of the dot containing $n$ electrons, respectively. The spacing between adjacent conductance peaks is given by the difference

$s_{n}=\mu_{n}-\mu_{n-1}=E_{G}(n+1)-2 E_{G}(n)+E_{G}(n-1)$.

Recently, a new generation of experiments on Coulomb blockade in ballistic and diffusive quantum dots has shown that the conductance peaks fluctuate both, with respect to their heights [9] and spacings [4] [7]. Most of the features of the peak height statistics are well understood within random matrix theory (RMT) [10]. However, all measured [4 7] peak spacing distributions resemble a Gaussian form, while the constant interaction model [8], which properly accounts for the average peak spacing, together with RMT predicts a Wigner-Dyson statistics. Moreover, the observed widths of the distributions vary between the experiments from a width comparable to the mean single-particle spacing $\Delta[6]$ to a width considerably larger [4, ], whereas the widths of the GOE and GUE distributions are about $\Delta / 2$. These experiments suggest that the widths scale rather with the charging energy $E_{c}$ than with $\Delta$.
Different theoretical studies addressed this issue: RPA diagrammatic perturbation theory yields corrections to the constant interaction predictions of order $1 / g$ [11] or $1 / \sqrt{g}$ [12], where $g$ is the dimensionless conductance. However, these approaches, which are valid at small $r_{s}$ (high densities), cannot easily explain the large widths $(\sim 8 \Delta)$ of the latest experiments [7]. A refined RMT approach [13], which accounts for shape deformations of the dot while adding electrons, explains the Gaussian profiles, while the widths remain comparable with $\Delta$.

Numerical exact diagonalization studies of an interacting tight-binding model of $\sim 10$ electrons show that for $r_{s}=1$ the distribution is indeed Gaussian [A]. This is also the situation when spin is included 14,15]. Nevertheless, questions were raised regarding the relevance of these calculations to the experimental system due to the small number of electrons considered, the use of a tightbinding model for which it is difficult to relate the parameters to the experimental systems, and due to the small values of $g$ (of order one) accessible in this approach.

In this paper we study interacting spinless electrons on continuous two-dimensional disordered cylinders at zero temperature. The interactions are considered within the self-consistent Hartree-Fock (SCHF) approximation, which enables us to treat up to $n=50$ electrons. By using a continuous model, the experimental parameters can directly be related to the calculations, and we obtain higher values of $g(g \sim 10)$ close to the experimental values. We show that within the SCHF approximation the peak spacing statistics is Gaussian in the presence or absence of magnetic field. The variance of the fluctuations is much larger than predicted in the independent-particle picture. This is in agreement with the exact diagonalisation results, with a density functional approach [14], and with other recent HF studies of disordered tight-binding models [16] and clean quantum dots [17]. Using Koopmans' theorem [18] we show that the calculated widths and Gaussian profiles can, at least partly, be related to 
specific Hartree interaction integrals (for not too strong interactions). We further show and explain that, on the contrary, the spacings among the occupied or the unoccupied HF levels follow GOE and GUE level statistics.

Numerical procedure - We begin with the HF equations for spinless electrons in real space,

$$
\begin{aligned}
& {\left[H_{0}+V_{\mathrm{dis}}(\vec{r})+\frac{e^{2}}{4 \pi \epsilon} \int_{\Omega^{\prime}} \frac{\sum_{j=1}^{n}\left|\psi_{j}\left(\overrightarrow{r^{\prime}}\right)\right|^{2}}{\left|\vec{r}-\overrightarrow{r^{\prime}}\right|} d \Omega^{\prime}\right] \psi_{k}(\vec{r})-} \\
& -\frac{e^{2}}{4 \pi \epsilon} \int_{\Omega^{\prime}} \frac{\sum_{j=1}^{n} \psi_{j}^{*}\left(\overrightarrow{r^{\prime}}\right) \psi_{k}\left(\overrightarrow{r^{\prime}}\right) \psi_{j}(\vec{r})}{\left|\vec{r}-\vec{r}^{\prime}\right|} d \Omega^{\prime}=\varepsilon_{k} \psi_{k}(\vec{r}) .
\end{aligned}
$$

Here $\epsilon$ is the dielectric constant, $H_{0}=-\left(\hbar^{2} / 2 m_{e}^{*}\right) \partial^{2} / \partial \vec{r}^{2}$ and $V_{\text {dis }}(\vec{r})=\sum_{q=1}^{N_{s}} \lambda_{q} \delta\left(\vec{r}-\vec{r}_{q}\right)$ the potential from $N_{s}$ $\delta$-scatterers at random locations $\vec{r}_{q}$ homogeneously distributed on the cylinder surface $\Omega$. The strengths $\lambda_{q}$ are chosen from a box distribution.

We use $0 \leq \theta \leq 2 \pi$ as polar and $0 \leq y \leq \pi$ as vertical coordinates and boundary conditions, $\psi_{j}(\theta, 0)=\psi_{j}(\theta, \pi)=$ $0, \psi_{j}(\theta+2 \pi, y)=e^{i 2 \pi \phi / \phi_{0}} \psi_{j}(\theta, y)$, to account for a flux $\phi$ piercing the cylinder. $\phi_{0} \equiv h c / e$ is the flux quantum. The set of Eqs. (2) is solved self-consistently by diagonalizing the Hamiltonian matrix in the basis of $H_{0}$. To this end we expand $\Psi_{j}(\vec{r}) \equiv e^{-i \theta \phi / \phi_{0}} \psi_{j}(\vec{r})=\sum_{l} a_{l}^{(j)} u_{l}(\vec{r})$ in the periodic basis functions $u_{l}(\vec{r})=(1 / \pi) e^{i n_{\theta} \theta} \sin \left(n_{y} y\right)$ of $H_{0}$, where $n_{\theta}=0, \pm 1, \pm 2, \ldots$ and $n_{y}=1,2,3 \ldots$ are good quantum numbers which define a level $l=l\left(n_{\theta}, n_{y}\right)$ of $H_{0}$. We emphasize that the enumeration of the levels $l\left(n_{\theta}, n_{y}\right)$ does depend on flux. Using energy units $\hbar^{2} /\left(m_{e}^{*} R^{2}\right)$, where $R$ is the cylinder radius, the matrix elements of the Hamiltonian (2) read

$H\left(l_{1}, l_{2}\right)=\varepsilon_{l_{1}}^{0} \delta_{l_{1}, l_{2}}+V_{\mathrm{dis}}\left(l_{1}, l_{2}\right)+U \sum_{l_{1}^{\prime}, l_{2}^{\prime}} A_{l_{1}^{\prime}, l_{2}^{\prime}} V_{l_{1}^{\prime}, l_{2}^{\prime}}\left(l_{1}, l_{2}\right)$

Here, $l, l_{i}, l_{i}^{\prime}$ denote eigenstates of $H_{0}$ and $\varepsilon_{l}^{0}=$ $1 / 2\left[\left(n_{\theta}+\phi / \phi_{0}\right)^{2}+\left(n_{y} / 2\right)^{2}\right]$ is the scaled energy of an electron on a clean cylinder of aspect ratio unity. $V_{\text {dis }}\left(l_{1}, l_{2}\right)$ are the disorder matrix elements. $U \equiv$ $R / 2 r_{B}$ determines the interaction strength in dimensionless units with $r_{B} \equiv 4 \pi \epsilon \hbar^{2} /\left(m_{e}^{*} e^{2}\right)$ the Bohr radius in the sample material. $A_{l_{1}^{\prime}, l_{2}^{\prime}}=\sum_{j=1}^{n} a_{l_{1}^{\prime}}^{*(j)} a_{l_{2}^{\prime}}^{(j)}$ and $V_{l_{1}^{\prime}, l_{2}^{\prime}}\left(l_{1}, l_{2}\right)=\left(l_{1}, l_{1}^{\prime}|v| l_{2}, l_{2}^{\prime}\right)-\left(l_{1}, l_{1}^{\prime}|v| l_{2}^{\prime}, l_{2}\right)$ with $\left.v \equiv 1 /\left|\vec{r}-\overrightarrow{r^{\prime}}\right|=\left[\sin ^{2}\left(\theta-\theta^{\prime}\right) / 2\right)+\left(y-y^{\prime}\right)^{2}\right]^{-1 / 2}$ in real space. While the matrix $A$ changes along the iterations until self-consistency is reached, $V_{\text {dis }}$ is calculated only once. We used $450 \times 450$ matrices and ensured that the results are practically unaffected by this truncation.

Generally, interaction effects are governed by the charge density $n_{s}$ and by $U$. We used $U=0.2 \pi^{2}=1.97$. On the one hand, this choice still allows convergence of the SCHF, on the other hand this is in the experimental parameter regime [4] 6]: In GaAs $r_{B}=10^{-2} \mu \mathrm{m}$ $\left(m_{e}^{*}=0.067 m_{e}, \epsilon=13 \epsilon_{0}\right)$. This gives for a typical dot area of $(2 \pi R)^{2} \sim 0.07-0.3 \mu \mathrm{m}^{2}$ the estimate $U \sim 2-4$ which is similar to the numerical value used. Accordingly, typical experimental values of $r_{s} \sim 1$ for GaAs [4 6] and $r_{s} \sim 2$ for Si [7] are also close to our numerical value $r_{s}=2 U \sqrt{\pi / n}$ that varies between 1.0 and 1.6 for $20 \leq n \leq 50$ electrons. The disorder strength used corresponds to $g \sim 10$ describing the experimental situation. $g$ was calculated from the inverse participation ratio [19].

Results and analysis - For a system containing $n$ electrons the SCHF ground state energy is given by 20

$E_{G}(n)=\sum_{k=1}^{n} \varepsilon_{k}-\frac{1}{2} \sum_{j, k=1}^{n}[(j, k|v| j, k)-(j, k|v| k, j)]$

where $j$ and $k$ enumerate SCHF levels and we redefined $v \equiv U /\left|\vec{r}-\overrightarrow{r^{\prime}}\right|$. Eq. (3) was used to calculate the peak spacings $s_{n}$ in Eq. (11). The peak spacing distributions were generated from an ensemble of 600 quantum dots (with different disorder realizations) and sequences of $s_{n}$ with $20<n<50$. The results are depicted as the histograms 1 in Fig. 1 for $\phi=0$ and Fig. 2 for finite $\phi$. The curves are centered around the mean peak spacing $\left\langle s_{n}\right\rangle \simeq 8.3 \Delta$. Clearly each distribution fits a Gaussian, curves 3 , defined by the average and variance of the data.

In the following we analyze the numerical results. Assuming that the single-particle states are unchanged by adding (subtracting) an electron to (from) the manyelectron system we can approximate the difference in energy of the ground states of the system with $n+1$ and $n$ electrons according to Koopmans [18] by

$$
\mu_{n} \equiv E_{G}(n+1)-E_{G}(n) \simeq \varepsilon_{n+1}(n) \text {. }
$$

Here, $\varepsilon_{n+1}(n)$ is the $n+1$ st energy eigenvalue in the system of $n \gg 1$ electrons. In view of Eq. (1), we then have approximately

$$
s_{n} \simeq \varepsilon_{n+1}(n)-\varepsilon_{n}(n-1) .
$$

The numerical distributions of the $s_{n}$ from Eq. (5) are depicted as curves 2 in Figs. 1 and 2. They show considerable agreement with the corresponding distributions (curves 1) of the SCHF ground state energies, Eq. (11), and their Gaussian fits. This shows that, for the interaction strengths considered, a peak spacing analysis based on Koopmans' relation is justified. As visualized in the right inset of Fig. 1, the peak spacing is related to energy differences from systems with different number of electrons [21]. We emphasize that $\varepsilon_{n}(n-1) \neq \varepsilon_{n}(n)$. Their difference is typically large ( $50 \%$ and more) on the scale of $s_{n}$ in Eq. (5). Hence $s_{n} \neq \varepsilon_{n+1}(n)-\varepsilon_{n}(n)$.

We also checked directly the validity of Koopmans' relation (4) by comparing the two numerical values for $\mu_{n}$ and $\epsilon_{n+1}(n)$. The typical relative error, the difference $\left(\varepsilon_{n+1}(n)-\mu_{n}\right) / \mu_{n}$, is always positive and $\sim 0.4 \%$, indicating the validity of Eq. (4). 


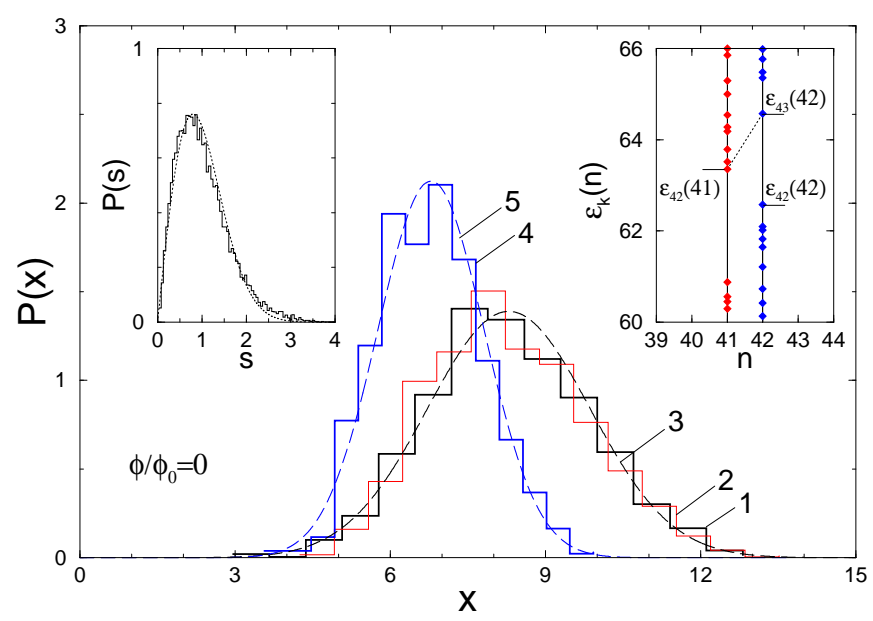

FIG. 1. Distributions of normalized Coulomb blockade peak spacings $x=s_{n} / \Delta$ for weakly disordered cylindrical quantum dots and flux $\phi=0$. The histograms 1 and 2 are calculated from the self-consistent Hartree-Fock ground state energies (Eq. (1)) and by using Koopmans' relation (5). Curve 3 is a Gaussian defined by the average (the charging energy) and variance of the data of curve 1. Curve 4 is the distribution of the exceptional direct integral (8) yielding a considerable contribution to $s_{n}$ in Eq. (d). Curve 5 is a Gaussian fit. Left inset: The nearest neighbour spacing distribution of SCHF single-particle energies for the occupied and unoccupied states is compared with the GOE statistics $p(s)=(\pi / 2) s \exp \left(-\pi s^{2} / 4\right)$ (dotted line). Right inset: A typical realization of SCHF eigenvalues. The dotted line connects eigenvalues relevant for calculating e.g., $s_{n=42}$ using Koopmans' relation (5).

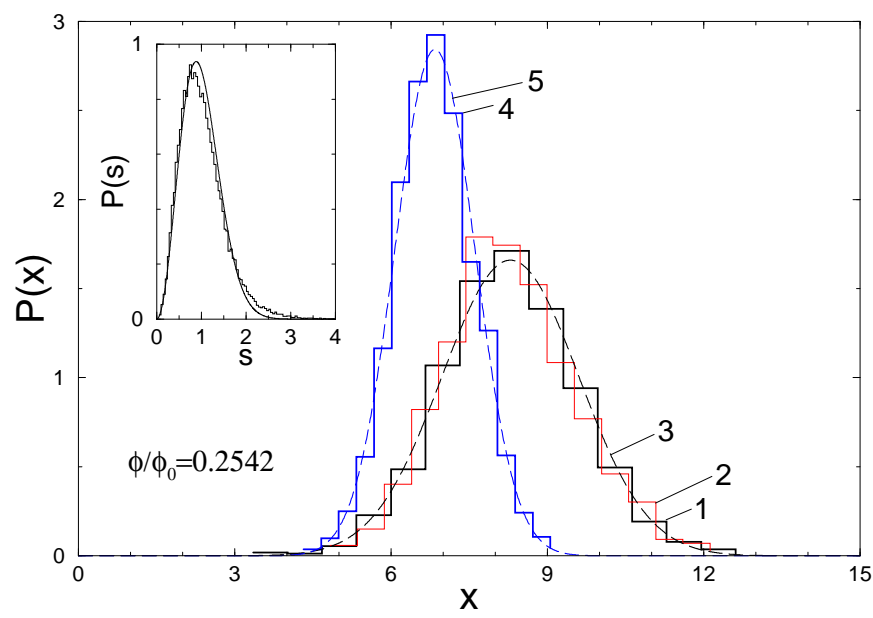

FIG. 2. SCHF peak spacing distributions as in Fig. 1 but for $\phi / \phi_{0} \simeq 0.25$ which reduces the fluctuations. Inset: The nearest neighbour distribution, as in the left inset of Fig. 1, of SCHF single-particle energies is compared with the GUE distribution $p(s)=\left(32 / \pi^{2}\right) s^{2} \exp \left(-4 s^{2} / \pi\right)$ (dotted line).

To understand the origin of the fluctuations of $s_{n}$ we recall that the HF energies $\varepsilon_{k}$ depend on $n$ and obey [20]

$\varepsilon_{k}(n)=\left(k\left|H_{0}+V_{d i s}\right| k\right)+\sum_{j=1}^{n}(j, k|v| j, k)-(j, k|v| k, j)$.
Using this relation in Eq. (5) we obtain

$$
\begin{aligned}
s_{n} & \sim\left[\left(n+1\left|H_{0}+V_{\text {dis }}\right| n+1\right)-\left(\tilde{n}\left|H_{0}+V_{\text {dis }}\right| \tilde{n}\right)\right] \\
+ & (n, n+1|v| n, n+1)-(n, n+1|v| n+1, n) \\
+ & \sum_{j=1}^{n-1}[(j, n+1|v| j, n+1)-(\tilde{j}, \tilde{n}|v| \tilde{j}, \tilde{n})] \\
& -\sum_{j=1}^{n-1}[(j, n+1|v| n+1, j)-(\tilde{j}, \tilde{n}|v| \tilde{n}, \tilde{j})] .
\end{aligned}
$$

The tilde sign denotes states calculated in the system with $n-1$ electrons. When $v \rightarrow 0$ one recovers the Wigner-Dyson distribution for $s_{n}$ which is modified upon increasing the interaction. The two sums in Eq. (7) contain differences between direct terms and between exchange terms which partly cancel each other. This is not the case for the direct and exchange integral in the second line which therefore represents an exceptional contribution to $s_{n}$. The direct term dominates over the exchange term because the integrand of the former is always positive. Hence we expect that the exceptional direct integral, $(n, n+1|v| n, n+1)$, describing the interaction between the $n$-th and $n+1$ st electron and given by

$$
U \int_{\Omega} \int_{\Omega^{\prime}} \frac{\left|\Psi_{n}(\vec{r})\right|^{2}\left|\Psi_{n+1}\left(\vec{r}^{\prime}\right)\right|^{2}}{\left|\vec{r}-\overrightarrow{r^{\prime}}\right|} d \Omega d \Omega^{\prime}
$$

significantly contributes to $s_{n}$.

It has been established 2223] that for moderate disorder the eigenfunctions fluctuate in space with small correlations. They even survive for different eigenfunctions but are weaker [24]. This should hold also in our SCHF calculations because the screening of the disorder potential is not perfect and disorder effects will persist. Because of the long-ranged Coulomb term, the central limit theorem should hold fairly good for the direct (Eq. (8)) and exchange integrals (despite the small wave functions correlations) and one thus expects to find a Gaussian distribution. For the interaction strengths used $(U \simeq 2)$ the direct term (8) can dominate the $s_{n}$ which implies a Gaussian distribution for the peak spacings, too.

In Figs. 1 and 2 the numerical distribution of the direct term (8) is shown as curve 4 and is compared with a Gaussian fit, curve 5. This distribution is expected to be centered to the left of the full numerical spacing distribution, because we have also to account for a shift on the scale of the mean HF single-particle spacing $\Delta_{H F}$ according to the first line in Eq. (7). Note that below the highest occupied and above the lowest unoccupied HF level, the spacing is $\Delta_{H F}=0.314>\Delta=0.183$. The shift to the left in Fig. 1 and Fig. 2 is $\sim 0.280 \sim 0.88 \Delta_{H F}$ and $\sim 0.270 \sim 0.84 \Delta_{H F}$, respectively.

Due to the appreciable interaction strength the discussion based on Eq.(7) is practically independent of flux. 
Both $s_{n}$ statistics are close to Gaussians but with fluxdependent widths.

Comparison with experiment - The Gaussian form of the distributions is in good agreement with all experimental results 田 [7]. The average peak spacing of the interacting systems is $\left\langle s_{n}\right\rangle / \Delta=1.52 / 0.183=8.27 \gg 1$ (curve 1 in Figs. 1 and 2), similar to the experimental situation in GaAs [4 6]. $\left\langle s_{n}\right\rangle$ is also in agreement with the estimated (scaled) classical capacitance of the cylinder, $\left[e^{2} / 4 \pi \epsilon(\pi R)\right] /\left[\hbar^{2} / m_{e}^{*} R^{2}\right]=0.4 \pi=1.26$

The width (RMS) of curve 1 in Fig. 1 (Fig. 2) equals $1.6 \Delta=0.19\left\langle s_{n}\right\rangle\left(1.3 \Delta=0.16\left\langle s_{n}\right\rangle\right)$. This is considerably larger than the widths $0.0966=0.52 \Delta(0.0743=0.41 \Delta)$ for the non-interacting case which are in good agreement with GOE (GUE) predictions [25].

The enhancement of the widths is compatible with the first GaAs experiments [4, [5] and in line with the Si experiment [7] which shows a larger width for higher values of $r_{s}$. The width is large compared to the experiment [6] where it was close to its non-interacting value. Since all experimental settings are quite similar, it is not clear what can lead to the different widths observed in [6].

SCHF single-particle statistics - We finally consider the statistics of the HF level spacings $s_{k}$ with $k \neq n$ to emphasize that they are not related to the Gaussian spacing distribution. By using Eq. (6) we have

$$
\begin{aligned}
s_{k} & =\varepsilon_{k+1}(n)-\varepsilon_{k}(n) \\
& =\left(k+1\left|H_{0}+V_{\text {dis }}\right| k+1\right)-\left(k\left|H_{0}+V_{\text {dis }}\right| k\right) \\
& +\sum_{j=1}^{n}[(j, k+1|v| j, k+1)-(j, k|v| j, k)] \\
& -\sum_{j=1}^{n}[(j, k+1|v| k+1, j)-(j, k|v| k, j)] .
\end{aligned}
$$

In contrast to Eq. (7) the exceptional and dominant direct integral does not appear in Eq. (9) leading to $s_{k} \ll s_{n}$. It is easy to see that for $k>n$ all terms contribute and for $k<n$ all terms except those with $j=k$ and $j=k+1$ that cancel each other. Hence there is no essential difference in the $s_{k}$ distributions in the two $k$ regions. Because of the tendency of the Coulomb terms to cancel each other, the terms in the second line in Eq. (9) are expected to dominate the average. The left insets of Figs. 1 and 2 demonstrate that the numerical distributions of the normalized $s_{k}$ follow indeed GOE and GUE statistics (dotted lines), respectively, showing the effect of time-reversal symmetry breaking. This RMT type of statistics is in line with other HF results that was found in a different type of experiment [26].

Conclusion - We have studied Coulomb blockade peak spacing fluctuations for interacting spinless electrons within the self-consistent Hartree-Fock approximation. The main features of the conductance peak spacing fluctuations are similar to the experimental ones. The
Gaussian distribution of the addition spectrum is interpreted as the result of the dominance of direct interaction terms governed by spatial fluctuations of the eigenfunctions. On the other hand the Hartree-Fock single-particle levels were shown to follow Wigner-Dyson statistics.

One of us, AC, would like to thank D. Bar-Moshe, A. Heinrich, S. Levit, D. Orgad, B. Shapiro and S. Shatz for valuable discussions. It would not have been possible to complete this work without crucial consultation in super computations by N. Fraenkel, G. Koren and J. Tal from the IUCC at Tel-Aviv University and S. Shatz from BIU.

[1] For recent reviews see, e.g., Mesoscopic Electron Transport, L. L. Sohn, L. P. Kouwenhoven, and G. Schön, eds., Nato ASI Series E 345, (Kluvers, Dordrecht, 1997).

[2] P. Maksym and T. Chakraborty, Phys. Rev. Lett. 65, 108 (1990).

[3] L.P. Kouwenhoven, T.H. Oosterkamp, M.W.S. Danoesastro, M. Eto, D.G. Austing, T. Honda, and S. Tarucha, Science, 28, 1788 (1997).

[4] U. Sivan, R. Berkovits, Y. Aloni, O. Prus, A. Auerbach and G. Ben-Yosef, Phys. Rev. Lett. 77, 1123 (1996).

[5] F. Simmel, T. Heinzel and D.A. Wharam, Europhys. Lett. 38, 123 (1997).

[6] S.R. Patel, S.M. Cronenwett, D.R. Stewart, A.G. Huibers, C.M. Marcus, C.I. Druöz, J.S. Harris Jr., K. Campman, and A.C. Gossard, Phys. Rev. Lett. 80, 4522 (1998).

[7] F. Simmel, D. Abusch-Magder, D.A. Wharam, M.A. Kastner, and J.P. Kotthaus (cond-mat 9901274).

[8] M.A. Kastner, Rev. Mod. Phys. 64, 849 (1992);

[9] A.M. Chang, H.U. Baranger, L.N. Pfeiffer, K.W. West, and T.Y. Chang, Phys. Rev. Lett. 76, 1695 (1996); J.A. Folk, S.R. Patel, S.F. Gogijn, A.G. Huibers, S.M. Cronenwett, C.M. Marcus, K. Campman, and A.C. Gossard, ibid, 76, 1699 (1996).

[10] R.A. Jalabert, A.D. Stone, and Y. Alhassid, Phys. Rev. Lett. 68, 3468 (1992).

[11] R. Berkovits and B.L. Altshuler, Phys. Rev. B 55, 5297 (1997).

[12] Y.M. Blanter, A.D. Mirlin, and B. Muzykantskii, Phys. Rev. Lett. 78, 24449 (1997).

[13] R. Vallejos, C.H. Lewenkopf and E.R. Mucciolo, Phys. Rev. Lett. 81, 677 (1998).

[14] M. Stopa, Physica B 251228 (1998).

[15] R. Berkovits, Phys. Rev. Lett. 81, 2128 (1998).

[16] S. Levit and D. Orgad, cond-mat/9901298; P.N. Walker, G. Montambaux, and Y. Gefen, cond-mat/9902258.

[17] K.H. Ahn, K. Richter, and I.H. Lee (unpublished).

[18] T. Koopmans, Physica 1, 104 (1935).

[19] V. N. Prigodin and B. L. Altshuler, Phys. Rev. Lett. 80, 1944 (1998).

[20] E.K.U. Gross, E. Runge and O. Heinonen, Many-Particle Theory, (Adam Hilger, 1991).

[21] This large spacing (right inset of Fig. 1) does not represent any gap in the excitation energies: The lowest excitation is given by $\varepsilon_{n+1}(n)-\varepsilon_{n}(n)-[(n, n+1|v| n, n+$ 1) $-(n, n+1|v| n+1, n)]$, which is of the order of $\Delta$. 
[22] M.V. Berry, J. Phys. A, 10, 2083 (1977).

[23] V.N. Prigodin, K.B. Efetov and S. Iida, Phys. Rev. Lett. 71, 1230 (1993); V.N. Prigodin, ibid 74, 1566 (1995).

[24] Y.M. Blanter and A.D. Mirlin, Phys. Rev. E, 55, 6514 (1997); A.D. Mirlin, (preprint) cond-mat/9712153.

[25] This is very similar to the exact diagonalization results
[4, 15] for which the fluctuations were substantially enhanced compared to the non-interacting case, and for $r_{s} \sim 1$ were of order of $15 \%$ of the average peak spacing. [26] U. Sivan, F.P. Milliken, K. Milkove, S. Rishton, Y. Lee, J.M. Hong, V. Boegli, D. Kern and M. DeFranza, Europhys. Lett. 25, 605 (1994). 